University of Vermont

UVM ScholarWorks

$1-1-2020$

\title{
A tandem evolutionary algorithm for identifying causal rules from complex data
}

John P. Hanley

University of Vermont

Donna M. Rizzo

University of Vermont

Jeffrey S. Buzas

University of Vermont

Margaret J. Eppstein

University of Vermont

Follow this and additional works at: https://scholarworks.uvm.edu/cemsfac

Part of the Human Ecology Commons, and the Medicine and Health Commons

\section{Recommended Citation}

Hanley JP, Rizzo DM, Buzas JS, Eppstein MJ. A tandem evolutionary algorithm for identifying causal rules from complex data. Evolutionary Computation. 2020 Mar;28(1):87-114.

This Article is brought to you for free and open access by the College of Engineering and Mathematical Sciences at UVM ScholarWorks. It has been accepted for inclusion in College of Engineering and Mathematical Sciences Faculty Publications by an authorized administrator of UVM ScholarWorks. For more information, please contact scholarworks@uvm.edu. 


\title{
A Tandem Evolutionary Algorithm for Identifying Causal Rules from Complex Data
}

\author{
John P. Hanley \\ jhanley@uvm.edu \\ Donna M. Rizzo \\ drizzo@uvm.edu \\ Department of Civil and Environmental Engineering, University of Vermont, \\ Burlington, 05405, USA
}

Jeffrey S. Buzas

Department of Mathematics and Statistics, University of Vermont,

Burlington, 05405, USA

Margaret J. Eppstein

maggie.eppstein@uvm.edu

Department of Computer Science, University of Vermont, Burlington, 05405, USA

https://doi.org/10.1162/evco_a_00252

\begin{abstract}
We propose a new evolutionary approach for discovering causal rules in complex classification problems from batch data. Key aspects include (a) the use of a hypergeometric probability mass function as a principled statistic for assessing fitness that quantifies the probability that the observed association between a given clause and target class is due to chance, taking into account the size of the dataset, the amount of missing data, and the distribution of outcome categories, (b) tandem age-layered evolutionary algorithms for evolving parsimonious archives of conjunctive clauses, and disjunctions of these conjunctions, each of which have probabilistically significant associations with outcome classes, and (c) separate archive bins for clauses of different orders, with dynamically adjusted order-specific thresholds. The method is validated on majority-on and multiplexer benchmark problems exhibiting various combinations of heterogeneity, epistasis, overlap, noise in class associations, missing data, extraneous features, and imbalanced classes. We also validate on a more realistic synthetic genome dataset with heterogeneity, epistasis, extraneous features, and noise. In all synthetic epistatic benchmarks, we consistently recover the true causal rule sets used to generate the data. Finally, we discuss an application to a complex real-world survey dataset designed to inform possible ecohealth interventions for Chagas disease.
\end{abstract}

Keywords

Evolutionary algorithm, epistasis, heterogeneity, multiplexer, learning classifier systems, machine learning.

\section{Introduction}

The causal rules underlying emergent properties of complex systems often exhibit heterogeneity, epistasis, and/or overlap. Empirical observations of such systems may be high-dimensional and typically include missing data, noise, and imbalanced classes. All of these complicate our ability to infer meaningful rule sets that map observed system features to outcomes of interest. We do not just seek "black box" association models with high prediction accuracy on a particular data sample. Rather, our primary intent is to develop a practical method for identifying parsimonious "white box," potentially 
causal, rule sets from data with these complexities. We aim to show that we can consistently recover the causal rule sets used to generate data samples in several benchmark problems with these complex characteristics. Succeeding in this task will increase confidence that we can identify potentially causal rule sets on messy real-world complex datasets. Such rule sets will not only provide robust prediction accuracy over different samples of the data, but importantly may also provide meaningful insights into causation, and thus inform interventions that could potentially change outcomes.

Heterogeneity exists when there are multiple underlying causes for the same outcome class. Evidence for heterogeneity exists in many systems, including bladder cancer (Urbanowicz et al., 2013), autism (Buxbaum et al., 2001), and American political parties (Poole and Rosenthal, 1984). Epistasis occurs when combinations of different feature values exhibit non-additive effects on outcomes, and is believed to be ubiquitous for many diseases (Moore, 2003), including breast cancer (Ritchie et al., 2001), blood pressure in rats (Rapp et al., 1998), and Behçet's disease (Kirino et al., 2013). Many systems exhibit both heterogeneity and epistasis. For example, different (i.e., heterogeneous) combinations of nonlinearly interacting (i.e., epistatic) transmission line outages (the features) can cause cascading failures that lead to the same patterns of power loss in the electrical grid (the outcome) (Eppstein and Hines, 2012). Similarly, the ecological niche of the American black bear (Ursus americanus) is epistatic, in that the species requires both a secluded area for denning and specific combinations of spring, summer, and autumn food sources (Larivière, 2001), and heterogeneous, because of the widely different combinations of denning and three-season diets that accommodate the bear population, contributing to a vast geographic range that spans from southern Mexico to northern Canada (Larivière, 2001). Furthermore, real-world datasets often include correlated features having significant overlap in heterogeneous explanatory rules, highly imbalanced classes (i.e., outcome classes that appear in different frequencies in the dataset), uncertainty (noise) in measured outcomes, and missing data (Hanley, 2017).

There are many practical applications that require an understanding of such complex relationships, such as the development of personalized drug therapies (Wilson, 2009), predicting consumer behaviors (Young Kim and Kim, 2004), identifying genegene and gene-environment causes for disease (Moore, 2003), and developing ecointerventions to reduce disease transmission in developing countries (Hanley, 2017). However, while the size and complexity of available datasets have recently exploded, computational tools for analyzing such systems have not kept pace (Wu et al., 2014).

Traditional statistical, data mining, and machine learning methods, such as analysis of variance (Wilson et al., 2017; Yousefi et al., 2016), logistic regression (Jarlenski et al., 2016; Li et al., 2016; Nesheli et al., 2016), and decision trees (Markellos et al., 2016; Nesheli et al., 2016) are well suited for univariate analysis of additive models. Some studies perform feature selection using univariate logistic regression models and then test higher-order interactions between the selected features (Kaplinski et al., 2015; Molina et al., 2015; Olivera et al., 2015). However, since these techniques rely on the presence of detectable univariate signals, they are not well-suited for epistatic problems where main effects are small or non-existent. For example, when using random forests (Breiman, 2001) with 10-fold cross-validation on the epistatic and heterogeneous synthetic genome dataset described in Section 3.3, we found that prediction accuracy averaged only about $55 \%$ for individual decision trees, but increased with the number of trees in the forest (plateauing at about 69\% with 500 trees, which is slightly higher than the prediction accuracy of the true causal rule set, implying overfitting); we were unable to recover any decision trees with the true causal rules. State-of-the-art classifiers using deep learning neural networks can also yield excellent class predictions from complex 
datasets (LeCun et al., 2015), but do not attempt to produce parsimonious causal rule sets.

Learning classifier systems (LCSs) are evolutionary algorithms (EAs) originally designed for real-time data assimilation in dynamically changing environments (Holland and Reitman, 1978), but have also been employed to analyze batch classification problems with epistatic, heterogeneous and/or overlapping rules (Urbanowicz and Moore, 2009). The most common type of LCS is the Michigan-style LCS, first introduced by Holland and Reitman (1978), which uses a genetic algorithm to evolve a population of classifiers (conjunctive clauses that each predict an outcome). Prediction is typically evaluated based on a weighted combination of all classifiers in the population, and fitness is based (at least in part) on the number of times a classifier correctly predicts the outcome of an input feature vector (Wilson, 1995). Michigan-style LCSs can be inefficient and subject to bias (based on sampling order) when applied to batch data. Pittsburgh-style LCSs, first introduced by Smith (1980), have also been applied to batch data by dividing the dataset into subsets, although it was reported that this dataset division can become problematic when a niche associated with an outcome is small (Franco et al., 2012). A Pittsburgh-style LCS evolves sets of classifiers as complete solutions (equivalent to disjunctions of conjunctive clauses).

After unsuccessful attempts to mine a real-world complex survey dataset on Chagas vector infestation (described in Subsection 3.5) using the Michigan-style LCS ExSTraCS 1.0 LCS (Urbanowicz et al., 2014), we introduced a new evolutionary approach to find heterogeneous and epistatic associations between input features and multiple outcome classes in large datasets (Hanley et al., 2016). In the current work, we further develop the method, show that it efficiently identifies the true causal rule sets in benchmark problems, and discuss application of the method in seeking potentially causal rule sets in messy real-world survey data, with important practical implications.

Our approach uses two EAs in tandem, each using an age-layered population structure (Hornby, 2006), and assesses fitness using a hypergeometric probability mass function (Kendall, 1952) that accounts for the size of the dataset, the amount of missing data, and the distribution of outcome categories. The first EA evolves an archive of conjunctive clauses (CCs) that have a high probability of a statistically significant association with a given outcome. The second EA evolves disjunctions of these archived CCs to create a parsimonious archive of probabilistically significant clauses in disjunctive normal form (DNF). See Appendix A in the Supplemental Materials (https: / www .mitpressjournals.org/doi/suppl/10.1162/evco_a_00252) for a brief discussion of differences between the current method and the method as originally presented in Hanley et al. (2016). Despite a similarity in names, our method is unrelated to the tandem evolutionary algorithm presented in Huang et al. (2007).

This article is organized as follows. In Section 2, we present our evolutionary approach and, in Section 3, we describe the test problems used. In Section 4, we show how the method efficiently finds the true causal models in the benchmark problems tested and show some results of the method on a complex real-world dataset related to Chagas disease infestation. We discuss our findings and compare them to published LCS results in Section 5 and summarize our contributions in Section 6.

\section{Proposed Evolutionary Algorithm}

We propose a system of two EAs in tandem, capable of mining large, heterogeneous datasets of $N$ feature vectors for possibly epistatic and heterogeneous associations between combinations of $L$ nominal, ordinal, and/or real-valued features that are possibly 
predictive of a given target class outcome $k$. The tandem algorithm decomposes the problem into first (a) evolving good conjunctive clauses and (b) subsequently evolving good disjunctions of these conjunctive clauses. This problem decomposition dramatically reduces the size of the search space, as will be shown in Section 4 .

The first EA (dubbed CCEA) evolves conjunctive clauses (CCs) of various orders, where the order is the number of features included in the clause; $1^{\text {st }}$-order clauses correspond to main (i.e., univariate) effects. The second EA (dubbed DNFEA) combines archived CCs with disjunctions to evolve clauses in disjunctive normal form (DNFs) of various orders, where the order is the number of CCs in a heterogeneous rule set; $1^{\text {st }}$-order DNFs comprise a single CC. The CCEA and DNFEA are run separately for each desired outcome class $k$ to identify a causal rule set.

Both the CCEA and the DNFEA are implemented using a customized version of the Age-Layered Population Structure (ALPS) algorithm (Hornby, 2006), as shown in Algorithm 1. Restricting competition by segregating into sub-populations by age, and periodically introducing new individuals into the lowest age layer, has been shown to be effective in preventing premature convergence (Hornby, 2006). In this study, we used 5 linearly-spaced nonarchive age-layers with an age gap of 3 between layers. In the CCEA, we maintain a maximum subpopulation of layersize $=L$ individuals; in the DNFEA we use layersize $=20$. In both the CCEA and the DNFEA, we maintain an additional 6th layer that serves as a parsimonious archive of probabilistically significant conjunctive clauses.

Pseudo code for the basic evolutionary algorithm (which is common to both the CCEA and DNFEA) is shown in Algorithm 1, and the tandem CCEA-DNFEA system is depicted graphically in Figure 1. We detail the fitness function in Subsection 2.1, the population model and high-level algorithm (common to both the CCEA and the DNFEA) in Subsection 2.2, and the genetic representations and variational operators (specific to the CCEA or DNFEA) in Subsection 2.3. Open source MATLAB code is available online (Hanley, 2019).

\subsection{Fitness Function}

For a given outcome class $k$, we define the Fitness of a given clause using a hypergeometric probability mass function (PMF) (Kendall, 1952), as follows:

$$
\text { Fitness (clause, } k)=\frac{\left(\begin{array}{c}
N_{k} \\
N_{\text {match }, k}
\end{array}\right)\left(\begin{array}{c}
N_{\text {tot }}-N_{k} \\
N_{\text {match }}-N_{\text {match }, k}
\end{array}\right)}{\left(\begin{array}{c}
N_{\text {tot }} \\
N_{\text {match }}
\end{array}\right)},
$$

where clause is a given CC (in the CCEA) or DNF (in the DNFEA); $N_{k}=$ the total number of input feature vectors associated with the target outcome class $k$ (e.g., presence or absence of a disease) without any missing values for any features present in the clause; $N_{\text {match }, k}=$ the number of input feature vectors for which the given clause is true and that have the target class $k$; $N_{\text {tot }}=$ the total number of input feature vectors without any missing values for any features present in the clause (regardless of the output class); and $N_{\text {match }}=$ the number of input feature vectors for which the clause is true (regardless of class).

Equation (1) is a powerful measure of fitness, since it quantifies the probability that the observed association between the clause and the target class $k$ is due to chance, taking into account the size of the dataset, the amount of missing data, and the distribution of outcome categories. We seek clauses with small values for Equation (1), because lower 


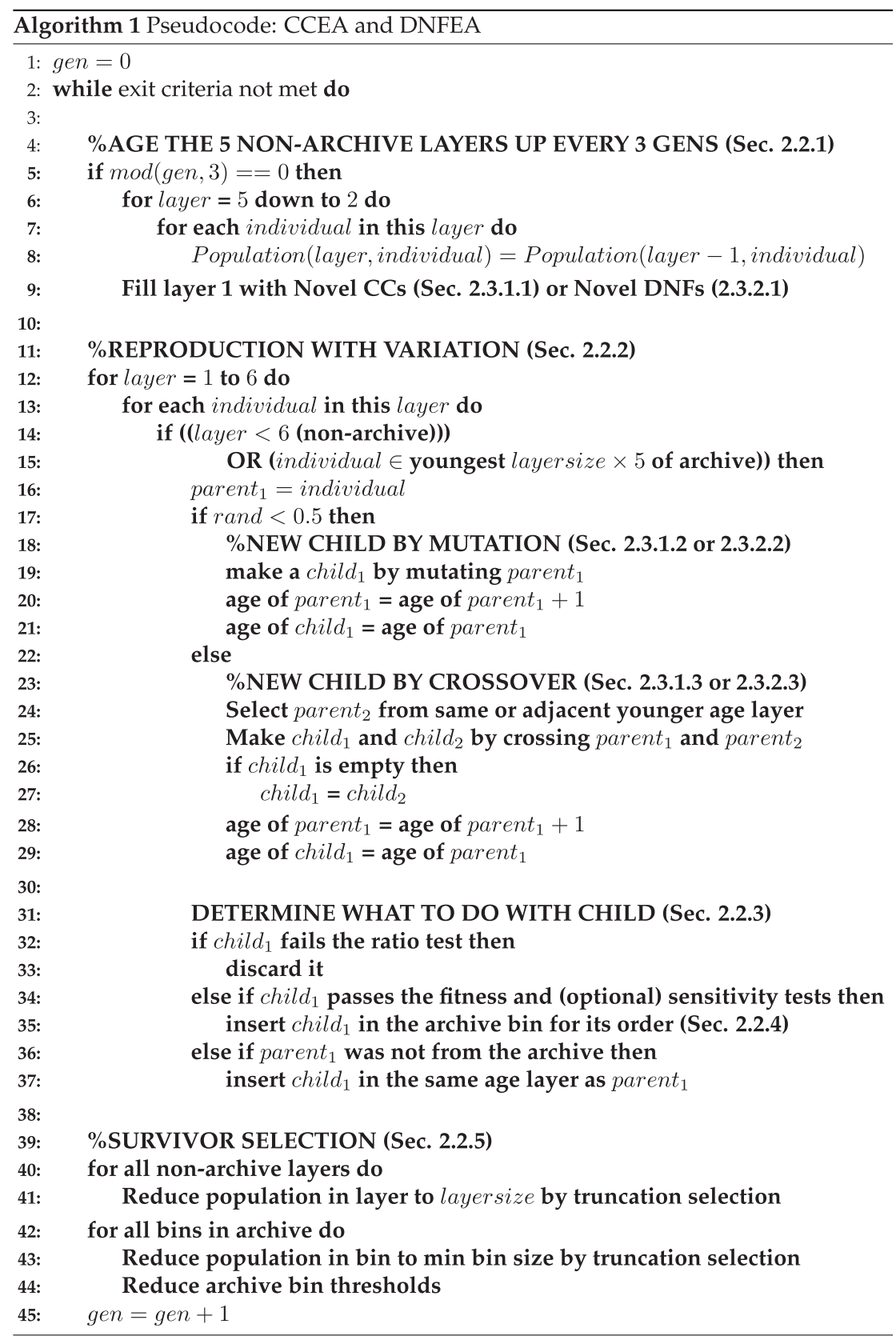

Fitness values are indicative of greater probability of association between a clause and a target class.

\subsection{Population Model}

\subsubsection{Initialization and Aging}

Initially, the population of clauses is empty. But as individuals are added, it is structured into subpopulations referred to as age layers, as a means of maintaining diversity 


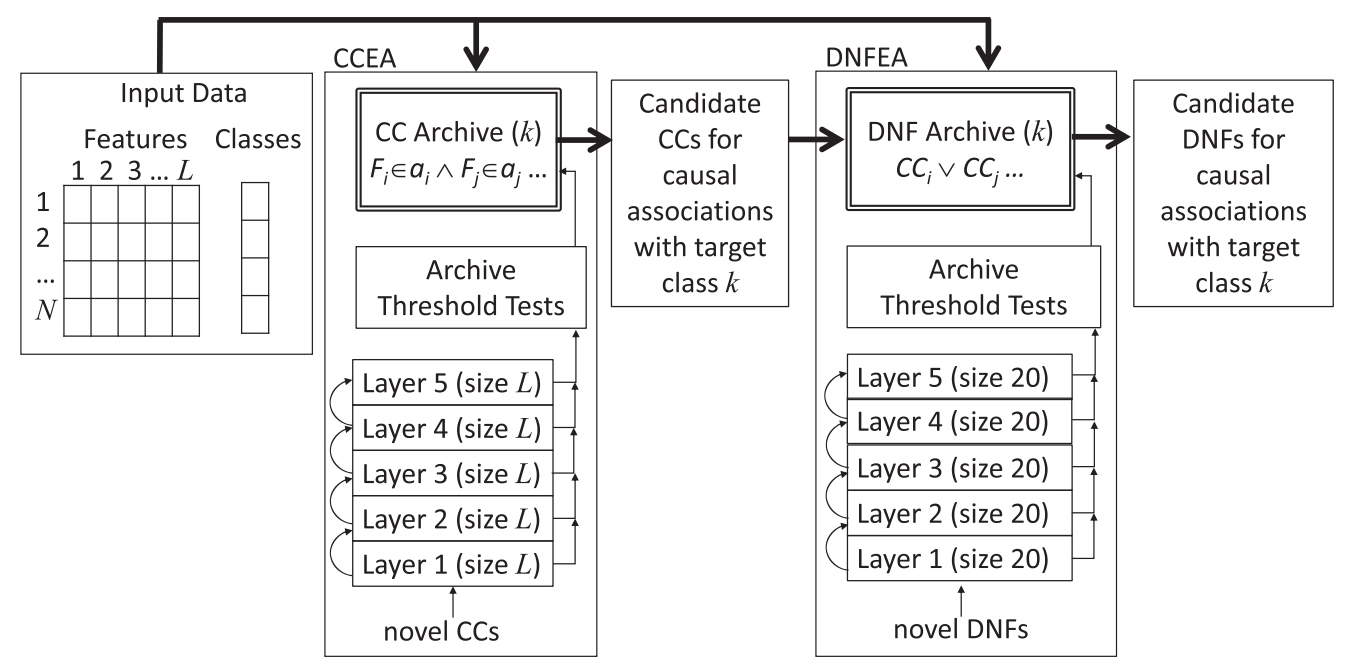

Figure 1: Graphical depiction of the proposed tandem ALPS-based EAs. For each target class $k$, we use the CCEA to evolve an archive of conjunctive clauses (CCs) that pass the threshold tests for archiving. The DNFEA then evolves disjunctions of these archived CCs and archives clauses in disjunctive normal form (DNFs) that pass the threshold tests for archiving.

(Hornby, 2006), with an age gap of 3 between layers. This means that every third generation, individuals in (non-empty) population layers 1-4 replace those in layers 2-5 (Alg. 1, lines 5-8) and a new random sub-population is created for layer 1, with the age of each novel clause initialized to 0 (Alg. 1, line 9). Further details of the initialization of CCs and DNFs are described in Subsections 2.3.1 and 2.3.2, respectively. Those aging out of layer 5 are discarded from the population.

\subsubsection{Reproduction}

During each generation, all individuals in layers $1-5$, plus the layersize $\times 5$ youngest individuals from layer 6 (the archive), are allowed to be parents (Alg. 1, lines 12-15). Each parent produces exactly one child through either mutation or crossover, with equal probability (Alg. 1, lines 17-29). If the child is to be produced by crossover, a second parent is selected from the same or preceding (if one exists) age layer, using tournament selection with replacement and a tournament size of 3. Details of the crossover and mutation operators on CCs and DNFs are described in Subsections 2.3.1 and 2.3.2, respectively. Each generation, the age of every individual that acted as a parent is incremented by 1 , and each child is given the age of its oldest parent (as in Hornby (2006)).

\subsubsection{Child Placement}

Every time a new clause is created, one of three things occur (Alg. 1, lines 31-37): (a) it is discarded, (b) it is added to the archive, or (c) it is added to the population layer of its oldest parent. Note: These steps are also carried out when new random children are created for layer 1 (Alg. 1, line 9). Three tests determine which of these events occurs:

- Ratio Test: If $\left(\frac{N_{\text {match }, k}}{N_{\text {match }}}<\frac{N_{k}}{N_{t o t}}\right)$, then the clause fails the ratio test. 
- Fitness Threshold Test: If Fitness (clause, $k) \leq T_{i}$, where $T_{i}$ is a dynamically adjusted order-specific fitness threshold for clauses of order $i$, then the clause passes the fitness threshold test.

- Optional Feature Sensitivity Threshold Test (CCs only): If Sensitivity $(C C, k)$ $\leq S_{i}$, where $S_{i}$ is a dynamically adjusted order-specific sensitivity threshold for clauses of order $i$, then the clause passes the feature sensitivity threshold test. Sensitivity $(C C, k)$ is defined as:

$$
\operatorname{Sensitivity}(C C, k)=\max _{\forall F_{i} \in C C}\left(\log _{10}\left(\frac{\operatorname{Fitness}(C C, k)}{\operatorname{Fitness}\left(C C-F_{i}, k\right)}\right)\right),
$$

where $F_{i}$ is a feature in the $C C$, and $C C-F_{i}$ represents the clause with that feature removed. Thus, Equation (2) calculates the maximum order of magnitude difference between the fitness of a given $\mathrm{CC}$ and the same $\mathrm{CC}$ with any one of its features removed (i.e., a lower-order, more general CC).

If a clause fails the ratio test, it is immediately discarded; this biases the algorithm toward retaining clauses that are useful in finding associations with the target class $k$. Otherwise if a DNF passes the fitness threshold test, or if a CC passes both the fitness threshold test and the optional feature sensitivity threshold test, then the clause is placed directly into the archive bin for order $i$ (unless a duplicate clause is already present). Using the additional feature sensitivity threshold test helps prevent archiving CCs with unnecessary features (i.e., overfitting the data) and also dramatically reduces the number of archived CCs and the consequent size of the DNFEA search space. The feature sensitivity threshold test is not used in the DNFEA, since the DNFEA only combines archived CCs that have already passed this test. If a retained clause is not archived and its oldest parent was not from the archive, it is inserted into the same population age-layer as its oldest parent, otherwise it is discarded.

\subsubsection{Archive Bins and Threshold Initialization}

Each archive is partitioned into bins for different clause orders $i$ to ensure diversity in the complexity of archived clauses. The maximum order and bounds on bin size are dataset-dependent (Tables C3-C5). The highest order bin may accept clauses with order $\geq i$.

All clauses in an archive bin for order $i$ have Fitness(clause, $k$ ) $\leq T_{i}$ (for both CCEA and DNFEA) and optionally also Sensitivity $(C C, k) \leq S_{i}$ (CCEA only). Thresholds $T_{i}$, for all orders $i$, are initialized to $1 / N$. This translates to an initial probability of 1 in $N$ that a clause with Fitness $=T_{i}$ is randomly associated with the target class $k$. In the CCEA, $S_{i}$ is similarly initialized to $\log _{10}(1 / N)$. These thresholds are dynamically reduced at the end of each generation (Subsection 2.2.5), so that the quality of the archived clauses increases as the evolution progresses.

\subsubsection{Survivor Selection and Dynamic Threshold Adjustment}

After reproduction, both nonarchive layers and archive bins may exceed their maximum allowed sizes, since new children have been added to the parent subpopulations. When this occurs, we use truncation selection to return the overflowing bins back to their minimum allowed sizes.

For each archive bin in both the CCEA and DNFEA, the fitness thresholds $T_{i}$ are dynamically reduced to the maximum Fitness value of all clauses remaining in bin $i$. If the feature sensitivity test is being used, we similarly reduce the feature sensitivity thresholds $S_{i}$ to the maximum value of Sensitivity, over all CCs remaining in bin $i$. 


\subsection{Representation of Clauses}

In this section, we describe the genetic representation of clauses and their variational operators, which are specific to the CCEA (Subsection 2.3.1) and the DNFEA (Subsection 2.3.2).

\subsubsection{Representation of Conjunctive Clauses (CCs)}

We represent possibly epistatic interactions that are predictive of a target class $k$, with CCs in the following form:

$$
C C_{k}:=F_{i} \in a_{i} \wedge F_{j} \in a_{j} \ldots,
$$

where $:=$ means "is defined as," $F_{i}$ represents a feature that may be nominal, ordinal, or continuous, and whose value lies in $a_{i}$, and $\wedge$ represents conjunction (i.e., logical AND). Note: $a_{i}$ is a set of values that is a proper nonempty subset of a prespecified universal set or maximum range of each feature in the dataset. The meaning of such a clause is interpreted as "if $C C_{k}$ is true for a given input feature vector, then the class outcome is predicted to be $k . "$

Any LCS classifier can be equivalently represented using the notation shown in Equation (3). For example, the LCS classifier 0\#\#1\# $\Rightarrow 1$ (where \# is a wild card symbol, which matches any value) is interpreted as "if feature 1 has value 0 and feature 4 has value 1 , then the outcome class is predicted to be 1 ." The condition $0 \# \# 1 \#$ is thus equivalent to the conjunctive clause $F_{1} \in\{0\} \wedge F_{4} \in\{1\}$ that is associated with outcome class 1. However, the notation in Equation (3) is more general, in that each feature $F_{i}$ can be of any data type, and $a_{i}$ can represent any set of values of that type.

Each CC is represented by two parallel data structures. The first is a Boolean vector of length $L$, where $L$ is the number of features in each input vector, that encodes presence (1) or absence (0) of each possible feature $F_{i}$ in the clause. Thus, the sum of the Boolean vector represents the order of the CC and each feature $i$ may appear at most once in a CC. When a feature is absent from a clause, it is equivalent to the LCS notation of having a wild card in that feature's position. We store the corresponding sets of values $a_{i}$ in a parallel data structure, as done in De Jong and Spears (1991). This is represented as a vector of $L$ pointers to binary masks indicating presence/absence of each value occurring for that feature anywhere in the entire dataset. If the data are ordinal or real-valued, we enforce all features indicated as present to have a contiguous range. Although this approach requires space proportional to the number of unique constants in the dataset, it affords constant-time checking to see if a given CC matches a given instance in the dataset. These parallel structures comprise the genome of an individual in the CCEA; the values in the binary vectors representing the presence/absence of feature $F_{i}$, and presence/absence of values in the corresponding set $a_{i}$, are coevolved.

We enforce at least one feature be present in each CC, and that the allowable set of values for each included feature be non-empty; this precludes the problem, discussed in Llorà et al. (2005), of evolving clauses that cannot match any instances in the dataset. We allow CCs to have up to $L$ features present, since we do not wish to make arbitrary $a$ priori assumptions on the maximum order of epistatic interactions that may exist and because, as shown in Iqbal et al. (2015), higher-order CCs can be useful in finding epistatic lower-order CCs.

2.3.1.1 CC Initialization. Novel CCs are randomly created for layer 1 to guarantee they match at least one input feature vector associated with the target class $k$, a process known as "covering" (Aguilar-Ruiz et al., 2003; Bacardit and Krasnogor, 2006, 2009; 
Franco et al., 2012). To accomplish this, we first generate a uniformly distributed random integer $j \in\{1, \ldots, L\}$ to specify the order of the CC, and then extract the subset of input feature vectors with class $k$ having at least this many nonmissing values. From this subset, we choose one at random. While the archive is empty, this random input feature vector is selected according to a uniform distribution. But once the archive has been populated with clauses, we use a nonuniform distribution to bias the selection toward input feature vectors not yet well-covered in the archive. Specifically, we first tally the archived clauses that match each input feature vector in the extracted subset. We then sum this tally, add one, subtract each feature vector's tally from this value, and cube the result (cubing increases the probability that under-represented input feature vectors will be selected). We normalize the resulting vector and treat this as the probability distribution, then select $j$ of the nonmissing features from the selected feature vector to be present in the new clause according to this distribution. For each selected feature $i$, we initialize $a_{i}$ to contain only the value for feature $i$ that occurs in the selected input feature vector.

2.3.1.2 CC Mutation. When a CC is selected for mutation, we do the following. Each position in a copy of the binary feature array from the parent is selected with probability $1 / L$ (if zero features were initially selected, we select one at random). For each feature $i$ selected, if the value at position $i$ in the binary feature array is 0 (feature is not present in the clause), then it is set to 1 (feature is added to the clause); and $a_{i}$ is randomly initialized to a nonempty set (for nominal features) or contiguous range (for ordinal or real-valued features) of allowable values that does not include the entire allowable subset or range of values. However, if the value at position $i$ in the binary feature array is 1 (i.e., $F_{i}$ was present in the clause), then with probability $P_{w}$, the bit is flipped to 0 (i.e., the feature is removed from the clause). We selected a high $P_{w}=0.75$ so mutation favors order reduction and helps evolve parsimonious clauses with as few features as possible. If the value at position $i$ in the binary feature array remains a 1 (feature $F_{i}$ is still present), the corresponding $a_{i}$ is mutated as follows. If $F_{i}$ is nominal, we randomly change, add, or delete a categorical value to $a_{i}$, ensuring the set remains non-empty and less than the allowable universal set of values. If $F_{i}$ is ordinal or continuous, we randomly change the lower or upper bound of $a_{i}$, ensuring the range remains non-empty, contiguous, and less than the maximum allowable range.

2.3.1.3 CC Crossover. When a CC is selected for crossover, we perform uniform crossover between copies of the CC and a mate selected as described in Subsection 2.2.2, where mate selection is based on Fitness. Specifically, we initially create two children, swapping values between random positions in the binary feature arrays of the two parent copies, and between the same positions in the corresponding value sets/ranges. If the first child contains at least one feature, we discard the second child; otherwise, we discard the first child.

\subsubsection{Representation of Clauses in Disjunctive Normal Form (DNFs)}

We represent possibly heterogeneous interactions that are predictive of a target class $k$ with DNFs in the following form:

$$
D N F_{k}:=C C_{i} \vee C C_{j} \ldots,
$$

where each $C C_{i}$ is of the form shown in Equation (3) and $\vee$ represents disjunction (i.e., logical OR). The meaning of such a clause is interpreted as "if $D N F_{k}$ is true for a given input feature vector, then the outcome class is predicted to be $k$." Each DNF is 
represented by a binary array of length $N_{C C, k}$, where $N_{C C, k}$ is the number of CCs archived by the CCEA for outcome class $k$. The binary values encode presence (1) or absence (0) of a given CC in the DNF, so the sum of this array represents the order of the DNF. Each DNF is constrained to include at least one CC but may have up to $N_{C C, k}$ CCs. This binary array comprises the genome of an individual in the DNFEA. For implementation efficiency, prior to running the DFNEA, each $C C$ in the archive is associated with a precomputed binary array of length $N$ that encodes whether the CC matches (1) or doesn't match (0) each of the $N$ input feature vectors and its associated outcome class, similar to the approach in Bacardit and Krasnogor $(2006,2009)$. In general, implementation of the DNFEA operators is simpler than that of the CCEA operators, since we no longer need to worry about allowable sets/ranges or covering of input feature vectors.

2.3.2.1 DNF Initialization. To create new DNFs for layer 1, novel DNFs are randomly created with anywhere from one CC to the maximum DNF order that will be archived for a given problem. If there are no DNFs in the archive, CCs are selected according to a uniform distribution. However, if there is at least one archived DNF, then CCs are selected by increasing the probability that a CC that covers more underrepresented input feature vectors with outcome class $k$ are selected for the initial DNF. We first follow the same steps described in Subsection 2.3.1.1 to create a probability distribution for selecting individual input feature vectors. We then create a vector with one element for each archived CC, where each element contains the sum of the probabilities of all input feature vectors covered by one archived CC. We normalize the resulting vector and treat this as the probability distribution for selecting a CC.

2.3.2.2 DNF Mutation. When a DNF is selected for mutation, it will undergo one of five mutation types with equal probability. Type 1 mutation is simple bit flip, where

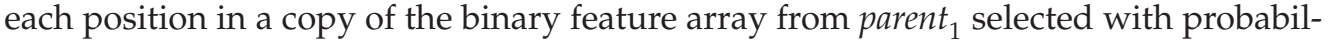
ity $1 / N_{C C, k}$ (if zero features were initially selected, we select one at random). We then perform bit-flip mutation at each of these selected positions, subject to the constraint that the DNF must still contain at least one CC.

The other four types of mutation are designed to expand the diversity of evolved clauses in terms of PPV and coverage, or are aimed at reducing the DNF order. Type 2 mutation adds the $\mathrm{C} C$ that covers the most target feature vectors not covered by parent ${ }_{1}$ (i.e., most likely to increase class coverage). Type 3 mutation adds the CC that covers the most target feature vectors not covered by parent $t_{1}$ while minimizing the number of new non-target feature vectors covered (i.e., will most likely improve Fitness). Type 4 mutation removes the $\mathrm{CC}$ that covers the least target feature vectors not covered by other CCs in the DNF (i.e., makes the DNF more parsimonious by sacrificing the smallest amount of class coverage). Finally, Type 5 mutation removes the $\mathrm{CC}$ that covers the most non-target feature vectors not covered by other CCs in the DNF (i.e., makes the DNF more parsimonious and also tries to increase the PPV). All five mutation types ensure that at least one CC will be present in the DNF.

2.3.2.3 DNF Crossover. When a DNF is selected for crossover, a mate is selected as described in Subsection 2.2.2, where mate selection is based on one of four criteria (used with equal probability): Type 1 selection picks the mate with the best Fitness. Type 2 selection picks the mate that covers the most target feature vectors not covered by parent (i.e., most likely to increase class coverage). Type 3 selection picks the mate that covers the most target feature vectors not covered by parent ${ }_{1}$ while minimizing the number 
Table 1: Challenges associated with benchmark problems used for testing; Majority-On (MO), Multiplexer (MP), 4 MP variants, synthetic Genome problem, and Chagas survey dataset.

\begin{tabular}{lccccccc}
\hline Problem & $\begin{array}{c}\text { Heter. } \\
\text { Rules }\end{array}$ & $\begin{array}{c}\text { Epistatic } \\
\text { Rules }\end{array}$ & $\begin{array}{c}\text { Overlap. } \\
\text { Rules }\end{array}$ & $\begin{array}{c}\text { Extran. } \\
\text { Features }\end{array}$ & $\begin{array}{c}\text { Imbal. } \\
\text { Classes }\end{array}$ & $\begin{array}{c}\text { Noisy } \\
\text { Classes }\end{array}$ & $\begin{array}{c}\text { Missing } \\
\text { Data }\end{array}$ \\
\hline MO & $\mathrm{X}$ & & $\mathrm{X}$ & & & & \\
MP & $\mathrm{X}$ & $\mathrm{X}$ & & & & & \\
MP V1 & $\mathrm{X}$ & $\mathrm{X}$ & & $\mathrm{X}$ & & & \\
MP V2 & $\mathrm{X}$ & $\mathrm{X}$ & & $\mathrm{X}$ & $\mathrm{X}$ & & \\
MP V3 & $\mathrm{X}$ & $\mathrm{X}$ & & $\mathrm{X}$ & & $\mathrm{X}$ & \\
MP V4 & $\mathrm{X}$ & $\mathrm{X}$ & & $\mathrm{X}$ & & & $\mathrm{X}$ \\
Genome & $\mathrm{X}$ & $\mathrm{X}$ & $\mathrm{X}$ & $\mathrm{X}$ & & $\mathrm{X}$ & \\
Chagas & $\mathrm{X}$ & $\mathrm{X}$ & $\mathrm{X}$ & $\mathrm{X}$ & $\mathrm{X}$ & $\mathrm{X}$ & $\mathrm{X}$ \\
\hline
\end{tabular}

of new nontarget feature vectors covered (i.e., will most likely improve the Fitness); and Type 4 picks the mate with the minimum nontarget feature vectors not covered by parent $_{1}$ (i.e., will most likely increase the PPV).

When a DNF is selected for crossover, we perform uniform or union crossover, with equal probability. For uniform crossover, if the first child contains at least one feature, we discard the second child; otherwise, we discard the first child. For union crossover, the child is created as the union of all CCs present in either parent.

\section{Test Problems}

The tandem CCEA and DNFEA were explicitly designed to search for parsimonious potentially causal rule sets in real-world problems that include heterogeneity, epistasis, overlap, and other complexities. However, in order to increase confidence on real-world applications, we tested the efficacy of an algorithm on benchmark problems with known causal rule sets. Unfortunately, few available benchmark problems exist in the literature with tunable heterogeneity, epistasis, and overlap, making it challenging to test the sensitivity of the algorithm to each of these features. While others have used k-DNF functions as benchmarks that include heterogeneity, epistasis, and overlap (Bacardit and Krasnogor, 2009; Franco et al., 2012; Calian and Bacardit, 2013), the random way in which these problems are generated does not allow systematic control on the degree of overlap or class imbalance. After spending significant time trying to generate custom benchmark problems (e.g., Hanley et al., 2016), we appreciate the difficulty in designing appropriate tunable benchmarks. Consequently, in this study we test the algorithm on three benchmarks previously used to test LCS algorithms. Two of these are classic scalable Boolean benchmark problems (the majority-on and multiplexer problems) and the third is a more realistic synthetic genome association problem (see Table 1). Finally, we apply our method to a real-world Chagas survey dataset.

In the majority-on problem (Subsection 3.1), individual features do not have unique meanings, unlike features in real-world classification problems. Yet despite known limitations (McDermott et al., 2012), we include some test results on this benchmark problem because it has maximum overlap that scales with the problem size and has been widely used as a challenging benchmark for Michigan-style LCS approaches (Iqbal et al., 2013b,c, 2014). 
The multiplexer problem (Subsection 3.2) is a standard benchmark problem that has tunable heterogeneity and epistasis, although no overlap; we created 4 additional multiplexer variants that include extraneous features, varying degrees of class imbalance, noise in class outcomes, and missing data (Table 1).

The majority-on and multiplexer problems help demonstrate how our proposed algorithm can recover the exact true causal rule sets under various degrees of controllable overlap, heterogeneity, and epistasis. We opted not to test on higher-order majority-on or multiplexer problems, or on the hybrid-parity multiplexer function from Butz et al. (2006), because the expected coverage of each of the individual true CCs in these problems is under $1.6 \%$, which is less than the heuristic $5 \%$ minimum coverage proposed in Bacardit and Krasnogor (2006) to prevent overfitting and certainly less than one would trust in an evolved solution to any real-world problem.

The synthetic genome problem (Subsection 3.3) was specifically designed to approximate a more realistic dataset that contains heterogeneity, epistasis, overlapping rules, extraneous features, and noise (Urbanowicz and Moore, 2010a).

An analysis of the entire CCEA search space evaluated on samples of input data for the majority-on, multiplexer, and synthetic genome problems (see Appendix B in the Supplemental Materials) illustrates (a) that there are many suboptimal clauses with PPV greater than or equal to the PPV of the true causal clauses (and in some cases greater coverage, as well), which underscores why PPV and coverage can be problematic fitness metrics for discovering the true causal CCs, (b) that the best Fitness varies between different orders of CCs for each given problem, which is why it is important to maintain order-specific thresholds for the Fitness bins in the CCEA and DNFEA archives, and (c) interesting fundamental differences in the structure of the fitness landscapes of the Boolean benchmark problems as compared to that of the more realistic synthetic genome problem (Fig. B1).

Finally, we apply the tandem evolutionary approach to the dataset that originally motivated the development of our algorithms. This messy real-world survey dataset was designed to identify features most associated with the infestation of Triatoma dimidi$a t a$, the vector that transmits the deadly Chagas disease, and includes correlated features, potentially extraneous features, imbalanced classes, noise in class labels, and missing data; previous research showed evidence that the causes of infestation are heterogeneous (Bustamante Zamora et al., 2015) and the root causes for infestation are suspected to be inherently epistatic. This Chagas dataset (described further in Subsection 3.5) thus includes many complexities (Table 1).

Each of these test problems is defined in more detail below.

\subsection{The Majority-On Problem}

In the majority-on problem, the number of input features $L$ is always odd and the outcome class is specified by which of the Boolean values ( 0 or 1$)$ is in the majority in a particular input feature vector. The causal rule set is the set of all classifiers with order $(L+1) / 2$ (see Table 2), such that all fixed bits and the action bit have the same value. For example, in the 3-bit majority-on problem, the causal rule set for outcome class 0 is the following disjunction: $(00 \#) \vee(0 \# 0) \vee(\# 00)$.

Since each condition may be considered a conjunctive clause (CC) (see Section 1 ), the causal rule sets may be considered in disjunctive normal form (DNF). Note: The causal rule sets are heterogeneous (since each is the disjunction of 3 classifiers). The classifiers are overlapping, yet not epistatic (i.e., all features have additive main effects). 
Table 2: Majority-On (MO) benchmark problem characteristics. The rightmost columns show expected change in class coverage and PPV when moving from a CC that is too general (e.g., 1\#\#) to the true higher-order CC that specifies one more feature (e.g., 11\#).

\begin{tabular}{|c|c|c|c|c|c|}
\hline Problem & $\begin{array}{l}\text { Order of } \\
\text { True CCs }\end{array}$ & $\begin{array}{l}\text { Order of } \\
\text { True DNF }\end{array}$ & $\begin{array}{c}\text { E[Coverage] } \\
\text { (per True CC) }\end{array}$ & $\begin{array}{c}\mathrm{E}[\Delta \text { Coverage }] \\
\text { (per } \uparrow C C \text { Order) }\end{array}$ & $\begin{array}{c}\mathrm{E}[\Delta \mathrm{PPV}] \\
\text { (per } \uparrow \mathrm{CC} \text { Order) }\end{array}$ \\
\hline 3-bit MO & 2 & 6 & $50.0 \%$ & $-25.0 \%$ & $25.0 \%$ \\
\hline 5-bit MO & 3 & 20 & $25.0 \%$ & $-18.8 \%$ & $12.5 \%$ \\
\hline 7-bit MO & 4 & 70 & $12.5 \%$ & $-10.9 \%$ & $6.3 \%$ \\
\hline 9-bit MO & 5 & 252 & $6.3 \%$ & $-5.9 \%$ & $3.1 \%$ \\
\hline 11-bit MO & 6 & 924 & $3.1 \%$ & $-3.0 \%$ & $1.6 \%$ \\
\hline
\end{tabular}

As the number of bits in the majority-on problem increases, the number of CCs in the true causal DNF increases exponentially (Table 2). For example, in the 11-bit problem there are 924 order-6 CCs in the true causal DNF. This explosion in rule set size occurs because the sensitivity of individual features drops dramatically as the majority-on problem size increases. For example, in the 3-bit problem, adding a single feature to an overly general CC (e.g., 1\#\#) to turn it into a true causal CC (e.g., 11\#) reduces the class coverage by $25 \%$ but increases the PPV by $25 \%$. However, in the 11 -bit problem, moving from an overly general CC (e.g., 11111\#\#\#\#\#\#) to a true causal CC (e.g., 111111\#\#\#\#\#) reduces class coverage by $3 \%$ but only increases the PPV by $1.6 \%$. Since the expected change in PPV (Table 2, rightmost column) due to the addition of a final true feature drops more rapidly than the expected class coverage (Table 2, second-to rightmost column), as a function of problem size, use of a feature sensitivity test for majority-on problems with more than 3-bits will necessarily fail. Consequently, we did not employ the optional feature sensitivity test in solving the majority-on problems.

\subsection{The Multiplexer Problem}

The multiplexer problem, designed to predict the output of an electronic multiplexer circuit, is another scalable Boolean benchmark problem. The multiplexer problem was first introduced to the machine learning community by Barto (1985), and has been a standard benchmark problem for testing LCS approaches for decades (Wilson, 1987a,b; Booker, 1989; Goldberg, 1989; De Jong and Spears, 1991; Butz et al., 2003, 2004, 2005; Bacardit and Krasnogor, 2006; Llorà et al., 2008; Bacardit and Krasnogor, 2009; Bacardit et al., 2009; Franco et al., 2011; Ioannides et al., 2011; Calian and Bacardit, 2013; Iqbal et al., 2012, 2013a,b,c, 2014, 2015; Urbanowicz and Moore, 2015).

The causal rule set is the disjunction of $2^{b+1}$ classifiers, each with order $b+1$, where $b$ is the total number of address bits used to identify a location in a vector of $2^{b}$ data bits that contains the outcome class. An example of the 6-bit multiplexer architecture is presented in Table C1 (see Appendix C in the Supplemental Materials). When using the multiplexer as a benchmark classifier problem, the input feature vectors comprise both the address bits and the data bits, so are $b+2^{b}$ bits long; the outcome classes associated with particular input feature vectors are thus only discovered as the address bits of the classifiers evolve. The causal rule set for outcome class 0 in the 6-bit multiplexer may thus be considered as the following DNF: $(000 \# \#) \vee(01 \# 0 \# \#) \vee(10 \# \# 0 \#) \vee(11 \# \# \# 0)$. This benchmark problem is purely epistatic (address features do not have main effects) 
Table 3: Multiplexer (MP) benchmark problem characteristics, where $\mathrm{E}$ is the expectation operator. The rightmost columns indicate the expected change in class coverage and PPV when moving from a CC that is too general (e.g., 0\#1\#\#\#) to the true higherorder CC that specifies one more feature (e.g., 001\#\#\#).

\begin{tabular}{lccccc}
\hline Problem & $\begin{array}{c}\text { Order of } \\
\text { True CCs }\end{array}$ & $\begin{array}{c}\text { Order of } \\
\text { True DNF }\end{array}$ & $\begin{array}{c}\text { E[Coverage] } \\
\text { (per True CC) }\end{array}$ & $\begin{array}{c}\mathrm{E}[\Delta \text { Coverage }] \\
\text { (per } \uparrow \text { Order) }\end{array}$ & $\begin{array}{c}\mathrm{E}[\Delta \mathrm{PPV}] \\
\text { (per } \uparrow \text { Order) }\end{array}$ \\
\hline 6-bit MP & 3 & 8 & $25.0 \%$ & $-12.5 \%$ & $25 \%$ \\
11-bit MP & 4 & 16 & $12.5 \%$ & $-6.3 \%$ & $25 \%$ \\
20-bit MP & 5 & 32 & $6.3 \%$ & $-3.1 \%$ & $25 \%$ \\
37-bit MP & 6 & 64 & $3.1 \%$ & $-1.6 \%$ & $25 \%$ \\
\hline
\end{tabular}

and heterogeneous (different classifiers match different subsets of the possible input vectors).

In the multiplexer problem, the number of CCs in the true causal DNF increases only linearly with the number of the problem bits (see Table 3). However, although the expected class coverage of a given true $\mathrm{CC}$, and the expected change in class coverage as the final true feature is added to a CC, are both halved as the number of bits in the problem is approximately doubled, we observe that the expected increase in PPV due to the addition of the final true feature remains constant at $25 \%$, regardless of problem size (Table 3). Thus, the feature sensitivity test is able to detect important features in the multiplexer problem.

We also report results for 4 variants of the 6-bit multiplexer problem with 14 extraneous features (with random binary values) added; (a) "Base Case": balanced classes, no noise in the output classes, and no missing data, (b) "Imbalanced": $85 \%$ class 0 and 15\% class 1, (c) "Noisy": 20\% noise in class outcomes (i.e., we flipped the outcome bit in $20 \%$ of random input data samples), and (d) "Missing data": $20 \%$ missing feature values (i.e., we randomly removed $20 \%$ of feature values from the input data samples).

\subsection{Synthetic Genome Problem}

Urbanowicz and Moore (2010a) designed a noisy dataset to represent a synthetic genome association study for a complex disease that incorporates both genetic epistasis and heterogeneity. For the remainder of this article, we refer to this as the synthetic genome problem. The dataset contains 1,600 input feature vectors, and is perfectly balanced in that 800 input feature vectors are associated with class 1 (disease) and 800 are associated with class 0 (no disease). Each input feature vector contains 20 ternary features, each representing whether a particular locus in the genome is homozygous for the major allele, heterozygous, or homozygous for the minor allele.

The dataset was designed with the intent that only four of these features would have a statistically meaningful association with the disease. Specifically, there were four heterogeneous causes for the simulated disease, in two pairs of purely epistatic interactions (i.e., no main effects) between two different pairs of loci (see Table 4). Since the association between each of these 4 causal rules and class 1 (disease) was designed to be noisy, we also indicate their PPV, coverage, and Fitness (Table 4).

Due to noise, the true causal DNF for class 1 (i.e., the disjunction of the 4 true causal rules shown in Table 4 ) has an overall PPV for class 1 of only $64 \%$ (see Table C2), coverage of $76 \%$, and Fitness of $3.2 \times 10^{-44}$. 
Table 4: The four causal rules designed to have a statistically meaningful association with class 1 (disease) in the synthetic genome problem. In each of the 4 rules, only two loci $\in\left\{F_{1}, F_{2}, F_{3}, F_{4}\right\}$ out of 20 are not wild cards. PPV, coverage, and Fitness of each of these true causal rules for disease are also shown.

\begin{tabular}{cccccccccccc}
\hline$F_{1}$ & $F_{2}$ & $F_{3}$ & $F_{4}$ & $F_{5}$ & $\ldots$ & $F_{20}$ & $N_{\text {match }}$ & $N_{\text {match }, k}$ & PPV & Cov. & Fitness \\
\hline 0 & 1 & $\#$ & $\#$ & $\#$ & $\ldots$ & $\#$ & 306 & 219 & $72 \%$ & $27 \%$ & $1.1 \times 10^{-17}$ \\
1 & 0 & $\#$ & $\#$ & $\#$ & $\ldots$ & $\#$ & 251 & 185 & $74 \%$ & $23 \%$ & $5.7 \times 10^{-17}$ \\
$\#$ & $\#$ & 0 & 1 & $\#$ & $\ldots$ & $\#$ & 334 & 222 & $66 \%$ & $28 \%$ & $4.2 \times 10^{-12}$ \\
$\#$ & $\#$ & 1 & 0 & $\#$ & $\ldots$ & $\#$ & 242 & 171 & $71 \%$ & $21 \%$ & $8.7 \times 10^{-13}$ \\
\hline
\end{tabular}

\subsection{Experimental Design for Benchmark Tests}

Control parameters for the different problem types and sizes tested are shown in Appendix Tables C3-C5, for the majority-on, multiplexer, and synthetic SNP problems, respectively. We note that, while preliminary experimentation showed these parameters were sufficient for identifying the true causal clauses, it is likely they could be further optimized to improve performance.

For the majority-on problem, the CCEA did not employ the feature sensitivity threshold, due to the pathological nature of this problem (described in Subsection 3.1); and we only report results for the DNFEA on the 3-bit and 5-bit majority-on problems. This is because, without a feature sensitivity threshold in the CCEA, the CC archive for the larger problems contained many archived CCs with orders greater than the order of the true CCs, and there was almost no difference between the PPV of the true CCs and those with higher order (i.e., those CCs that overfit the data).

In each of the benchmark problems, the 2 outcome classes are mutually exclusive; thus, we evolve rule sets to predict class 1, and make the assumption that "if class 1 is not predicted, then predict class 0," as in Bacardit et al. (2009); Bacardit and Krasnogor (2009); Franco et al. (2012). Runs were terminated when all of the true causal clauses had been archived; we recorded the total number of fitness evaluations performed per run. Each problem was run for 30 random repetitions. The number of random training input feature vectors for the majority-on and multiplexer problems was dependent on the problem size (Tables C3-C4). The synthetic genome problem was trained on the 1,600 input feature vectors provided by Urbanowicz and Moore (2010a).

\subsection{Chagas Vector Infestation Dataset}

Through a collaborative effort between the University of Vermont, Loyola University New Orleans, and La Universidad de San Carlos Guatemala, we performed detailed socioeconomic and entomological surveys on over 20 towns in Guatemala, El Salvador, and Honduras to study the risk of infestation with T. dimidiata, the vector for the deadly Chagas disease (Hanley, 2017). The surveys contain 64 risk factors that experts believe may be associated with infestation of households. Seven of the risk factors are ordinal, six are integer, 1 is continuous, and the remaining 50 are nominal. The dataset comprises 311 input feature vectors of length 64 , has $26 \%$ missing data, and imbalanced class outcomes with 100 (32\%) infested households. Because of the large number of features, we selected relatively large CC archive bins (250 to 260 CCs per order). Since we are interested in lower-order DNFs in this application, we restricted the DNF archive to order 10 or less (100 to 110 DNFs per order). 
Table 5: Summary of Majority-On results with no feature sensitivity test. Values represent the median over 30 repetitions.

\begin{tabular}{lccccc}
\hline Majority-On & 3-bit & 5-bit & 7-bit & 9-bit & 11-bit \\
\hline CCEA Space $^{1}$ & 52 & 484 & 4,372 & 39,364 & 354,292 \\
\#CCEA Evals $^{2}$ & 34 & 323 & 1,974 & 11,924 & 97,700 \\
\#Archived CCs $^{3}$ & 9 & 42 & 147 & 585 & 5,745 \\
DNFEA Space $^{4}$ & 372 & $1.7 \times 10^{10}$ & $N A$ & $N A$ & $N A$ \\
\#DNFEA Evals $^{5}$ & 255 & 278,818 & $N A$ & $N A$ & $N A$ \\
\#Archived DNFs $^{6}$ & 53 & 164 & $N A$ & $N A$ & $N A$ \\
\#Total Evals $^{7}$ & 294 & 279,307 & $N A$ & $N A$ & $N A$ \\
De novo DNF Space $^{8}$ & $2.9 \times 10^{6}$ & $3.1 \times 10^{23}$ & $N A$ & $N A$ & $N A$ \\
\hline
\end{tabular}

\footnotetext{
${ }^{1}$ Maximum number of CCs from all possible features.

${ }^{2}$ Median number of CCEA fitness evaluations.

${ }^{3}$ Number of CCs in the CCEA archive searched by the DNFEA.

${ }^{4}$ Maximum number of DNFs from median number of archived CCs.

${ }^{5}$ Median number of DNFEA fitness evaluations.

${ }^{6}$ Median number of DNFs in the DNFEA archive.

${ }^{7}$ \#CCEA Evals + \#DNFEA Evals.

${ }^{8}$ Number of possible DNFs if using all possible CCs.
}

\section{Results}

\subsection{Results on Majority-On Problems}

For the majority-on problems, the CCEA archived all of the true causal CCs in all 30 runs for each the 3-bit to 11-bit problems. However, the CCEA search process was only slightly more efficient than exhaustive search (compare the \#CCEA Evals to CCEA space in Table 5). This is because these problems have relatively small search spaces and a relatively large number of true causal CCs. In such problems, it may be reasonable to simply perform an exhaustive search to find all the true CCs.

The DNFEA was very efficient in finding and archiving the true causal DNF in all 30 runs for each of the 3-bit and 5-bit majority-on problems (see Table 5). In the 5-bit problem, for example, the DNFEA required 5 orders of magnitude fewer evaluations than exhaustive search of the CC archive (the DNFEA Space), and 18 orders of magnitude fewer evaluations than if searching all possible CCs (the so-called De novo DNF space in Table 5). As mentioned in Subsection 3.4, the CCEA archives were too large for the 7-bit to 11-bit majority-on problems; and the differences in the PPVs of these archived CCs were too low for effective application of the DNFEA. Note: We were successful in using the DNFEA to recover the true causal rule sets in 30 repetitions of each of the 7-bit to 11-bit majority-on problems if we post-processed the CC archive to retain only CCs with orders less than or equal to the order of the true CCs, and with PPV of $100 \%$. Since this rather ad hoc postprocessing of the archive required some foreknowledge of the problem solution, we have elected not to present these results.

\subsection{Results on Multiplexer Problems}

On all multiplexer problems, the tandem CCEA and DNFEA always archived the true causal rule sets for all 30 repetitions of all problem sizes; and in nearly all cases, the true causal DNF was readily identifiable as the archived solution with the best Fitness (the only exception being when there was $20 \%$ missing data, discussed in more detail below). 
Table 6: Summary of Multiplexer results. Values represent the median over 30 seeded repetitions. Rows are the same as in Table 5.

\begin{tabular}{lcccc}
\hline Multiplexer & 6-bit & 11-bit & 20-bit & 37-bit \\
\hline CCEA Space & 1,456 & 354,292 & $7.0 \times 10^{9}$ & $9.0 \times 10^{17}$ \\
\#CCEA Evals & 544 & 4,685 & 39,997 & 307,206 \\
\#Archived CCs & 16 & 73 & 230 & 661 \\
DNFEA Space & $1.5 \times 10^{4}$ & $7.3 \times 10^{11}$ & $2.8 \times 10^{26}$ & $1.2 \times 10^{57}$ \\
\#DNFEA Evals & 561 & 4,275 & 23,501 & 125,380 \\
\#Archived DNFs & 69 & 131 & 251 & 478 \\
\#Total Evals & 1,121 & 9,331 & 62,313 & 434,215 \\
De novo DNF Space & $1.3 \times 10^{16}$ & $8.6 \times 10^{48}$ & $2.4 \times 10^{161}$ & $\left(\begin{array}{c}9.0 \times 10^{17} \\
34\end{array}\right)$ \\
\hline
\end{tabular}

Table 7: Summary of 6-bit Multiplexer results with 14 extraneous features added in the Base Case along with either Imbalanced classes (15\% class 1), Noisy outcomes (with 20\% random errors), or 20\% Missing Data. Values are the median over 30 seeded repetitions. Rows are the same as in Table 5.

\begin{tabular}{lcccc}
\hline Multiplexer V1-V4 & Base Case & Imbalanced & Noisy & Missing Data \\
\hline CCEA Space & $7.0 \times 10^{9}$ & $7.0 \times 10^{9}$ & $7.0 \times 10^{9}$ & $7.0 \times 10^{9}$ \\
\#CCEA Evals & 3,523 & 3,445 & 3,230 & 3,914 \\
\#Archived CCs & 26 & 15 & 25 & 20 \\
DNFEA Space & 313,885 & 9,933 & 245,480 & 60,439 \\
\#DNFEA Evals & 813 & 582 & 1,071 & 1,380 \\
\#Archived DNFs & 68 & 68 & 69 & 69 \\
\#Total Evals & 4,296 & 4,145 & 4,747 & 5,168 \\
De novo DNF Space & $2.4 \times 10^{161}$ & $2.4 \times 10^{161}$ & $2.4 \times 10^{161}$ & $2.4 \times 10^{161}$ \\
\hline
\end{tabular}

The tandem search algorithm required many orders of magnitude fewer evaluations than using exhaustive search (compare the \#Total Evals to the size of the De novo DNF Space in Tables 6 and 7), and the relative efficiency of the method improved dramatically as the problem size increased.

The method is robust to the inclusion of extraneous features, imbalanced classes, noisy classes, and missing data. For example, in Figure 2 a we show results from a typical 6-bit multiplexer run with 14 extraneous features. In this figure, archived CCs are shown with green squares, where darker shading indicates higher-order conjunctions. Similarly, archived DNFs are shown with blue circles, where darker blue indicates higher-order disjunctions. For clarity, the four true archived 3rd order causal CCs are shown in orange hexagrams and the single true archived 4th order causal DNF for class 1 is shown with the red pentagram. In this noise-free problem the true DNF is clearly identifiable as the single solution that has the best Fitness. Even with highly imbalanced classes $(85 \% / 15 \%)$, the tandem algorithm is able to reliably find the exact causal DNF for the minor class (e.g., Fig. 2b). When $20 \%$ noise is added to the outcome classes, the PPV and coverage are necessarily reduced, but the true causal DNF still consistently 


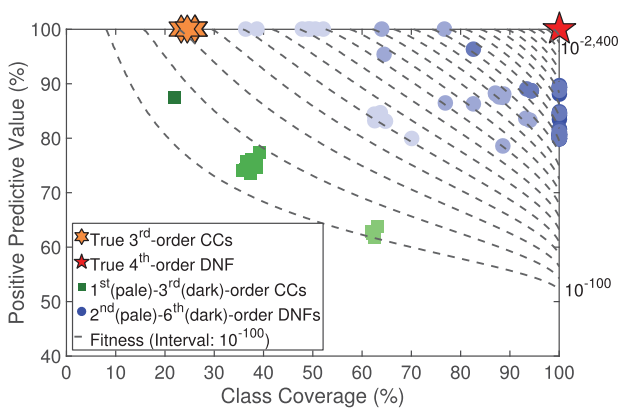

(a)

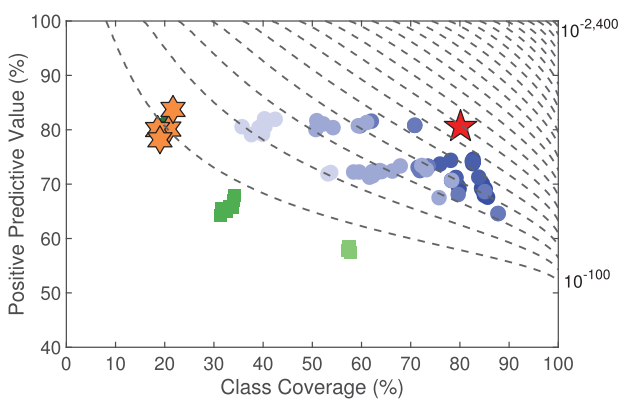

(c)

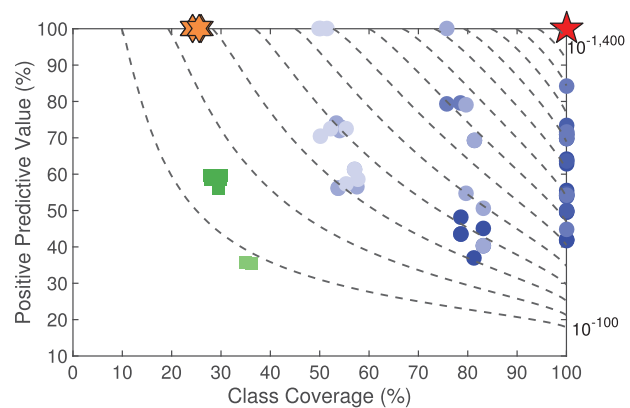

(b)

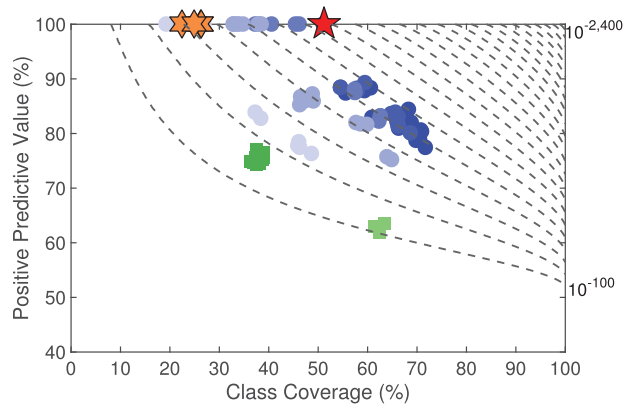

(d)

Figure 2: Typical archived results (arbitrarily selected as the first of 30 repetitions) using 8,000 random training instances for target class 1 on the 6-bit multiplexer problem with 14 extraneous features added and (a) balanced classes with no noise or missing data; or with either (b) imbalanced class outcomes (15\% class 1 ); (c) $20 \%$ random errors in class outcome; or (d) 20\% randomly missing feature data. The contour lines represent equally spaced values of Fitness.

stands out as the archived DNF with the best Fitness (e.g., Fig. 2c). Finally, we observed that even with $20 \%$ missing data in the input dataset, the true causal DNF always had orders of magnitude better fitness than any other 4th-order DNF (e.g., Fig. 2d), although in some runs there were a few (median $=7$ over the 30 repetitions) 5 th and 6 th order clauses that had slightly better fitness. In these cases, the true causal DNF could still be identified as the most parsimonious (i.e., lowest order) of the highly fit DNFs.

\subsection{Results on Synthetic Genome Problem}

The synthetic genome problem includes extraneous features and noise in class outcomes, so it is not possible to achieve $100 \%$ PPV or coverage. However, all 4 true causal 2nd-order CCs were consistently archived in all 30 repetitions. The true causal 4 th-order DNF was also archived in all 30 trials. We found numerous DNFs with higher PPV than the true causal DNF and a few other higher-order DNFs with similar or slightly better Fitness; however, the true causal DNF stands out as the most parsimonious (lowest order) of the fittest DNFs in the archive, and as the archived DNF with the highest coverage (see Figure 3).

The synthetic genome problem highlights the importance of using the feature sensitivity test to filter the number of CCs added to the CCEA archive. There are 20 features in the synthetic genome problem, and only 4 are contained in the true causal rule set. 


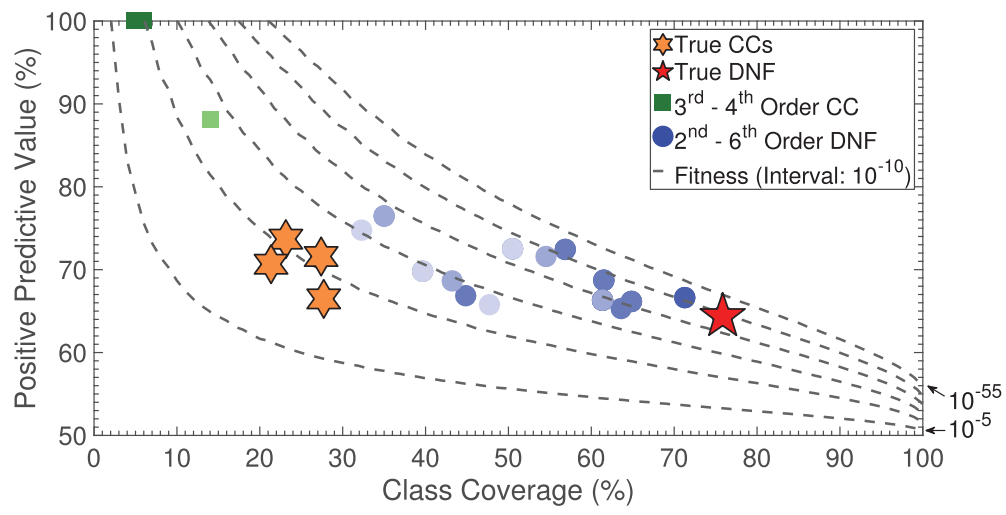

Figure 3: Archived results on the Synthetic Genome Problem (arbitrarily selected as the first of 30 repetitions) using the 1,600 training instances. The contour lines represent equally spaced values of Fitness.

Table 8: Summary of results on the synthetic genome problem. All values are the median over 30 repetitions. The feature sensitivity test was used in the CCEA for all runs. Rows are the same as in Table 5.

\begin{tabular}{lc}
\hline Synthetic Genome & Medians \\
\hline CCEA Space & $1.1 \times 10^{12}$ \\
\#CCEA Evals & 3,529 \\
\#Archived CCs & 7 \\
DNFEA Space & 119 \\
\#DNFEA Evals & 106 \\
\#Archived DNFs & 40 \\
\#Total Evals & 3,595 \\
De novo DNF Space & $2.5 \times 10^{69}$ \\
\hline
\end{tabular}

Nonetheless, there are thousands of potential CCs that pass the initial ratio and fitness threshold tests for the archive. However, there are only 11 possible CCs that would also pass the feature sensitivity test. In practice, adding the feature sensitivity filter reduced the median number of archived CCs to 7 (see Table 8). With so few CCs archived, the DNFEA required only slightly fewer fitness evaluations than exhaustive search (compare the \#DNFEA Evals to the size of the DNFEA Space in Table 8).

\subsection{Results on Chagas Vector Infestation Dataset}

The CCEA archived 1,089 CCs (Fig. 4), and discovered several interesting heterogeneous and overlapping CCs (ranging from main effects through 7th-order epistatic CCs). On this real-world problem, incorporating the feature sensitivity threshold reduced the maximum order of archived clauses from 24th-order to 7th-order, thus removing thousands of the very high order CCs most likely the product of overfitting.

The DNFEA archived 940 probabilistically-significant DNFs (see Figure 4). In this real-world dataset, it is unlikely that a single causal DNF exists. Due to feature 


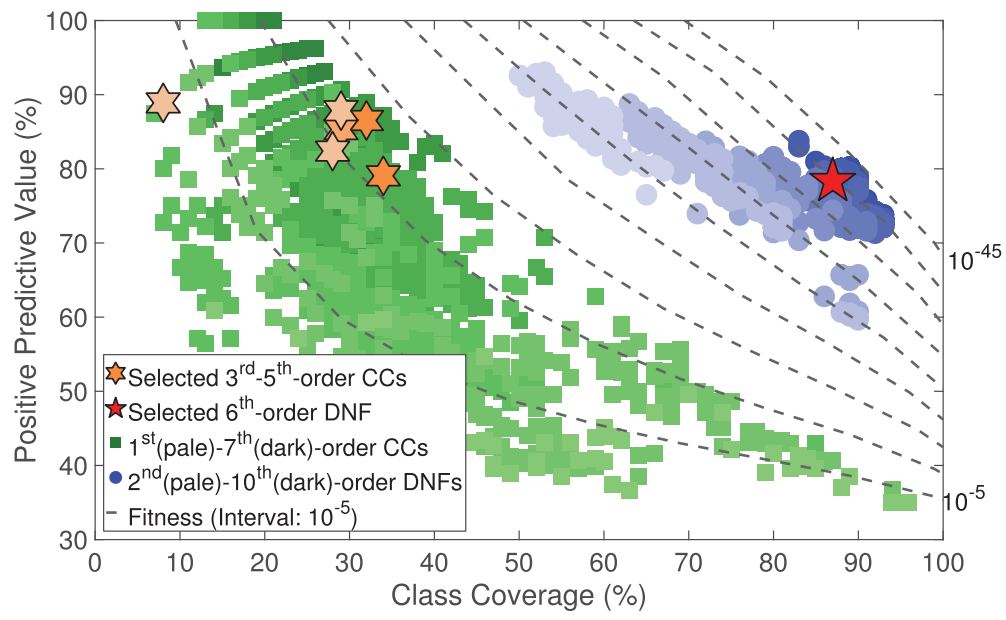

Figure 4: Archived Chagas survey results. Axes represent the PPV and class coverage on the 311 training instances, 100 of which are associated with infestation. Contours represent equally spaced Fitness. The pentagram and hexagrams are selected candidates for possibly causal rule sets.

correlations, missing and noisy data, there are many rule sets with very strong statistical associations with infestation of the Chagas vector. We are also well aware of the risk of overfitting in this relatively small dataset; so we do not claim to have "solved" the problem. Figure (4) highlights one of many interesting candidate DNFs, selected as the most parsimonious of the highly fit DNFs with Fitness $<10^{-39}$. This 6th-order DNF has Fitness $=4 \times 10^{-40}, \mathrm{PPV}=78 \%$, Coverage $=87 \%$, and comprises CCs ranging from order 3 to order 5 (also highlighted in Fig. 4), whose coverage sums to 152\%, indicating overlap. This and other highly fit DNFs are interesting candidates solutions that may provide insight into the primary drivers of T. dimidiata infestation.

\section{Discussion}

To tackle the challenge of seeking potentially causal rules sets for explaining complex real-world data, we propose a new approach using tandem age-layered evolutionary algorithms on batch data. In this section, we discuss some key aspects of the CCEADNFEA algorithm, compare our results on benchmark problems to published LCS results, and briefly discuss the application of the method to a real-world survey problem.

\subsection{Hypergeometric PMF as a Fitness Metric}

We propose using the hypergeometric PMF (Eq. (1)) as a principled statistic rooted in probability theory (Kendall, 1952) for assessing relative fitness of clauses of a given order in complex classification problems. Unlike other fitness functions that incorporate ad hoc weighted sums and/or products of PPV, coverage, and/or model complexity (Aguilar-Ruiz et al., 2003; Llorà et al., 2008; Bacardit et al., 2009), the hypergeometric PMF quantifies the probability that the observed association between a given clause and target class is due to chance, taking into account the size of the dataset, the amount of missing data, and the distribution of outcome categories. 
We use dynamically-adjusted, order-specific, probability thresholds to determine which CC and DNF clauses to archive. Of course, while these clauses are potentially causal, a low probability that the association is by chance does not in itself imply causation (Nuzzo, 2014). Equation (1) enables our algorithm to archive parsimonious clauses with different combinations of PPV and coverage while gracefully handling imbalanced classes, missing data, and noisy class associations.

\subsection{CCEA and DNFEA}

The CCEA creates an archive of CCs, each with a probabilistically significant association with a given outcome class. The DNFEA subsequently creates an archive of probabilistically significant disjunctions of the archived CCs. As in Hornby (2006), we found the age-layering to be very important in maintaining diversity, which facilitated continual improvement over the evolutionary process.

By maintaining separate archive bins for clauses of different orders, and using a feature sensitivity threshold to filter the CCs with unwarranted complexity, the tandem algorithm evolves parsimonious rule sets without a priori assumptions on the maximum order of interactions (as in Urbanowicz and Moore, 2015) or the inclusion of ad hoc penalty terms in the fitness function (as in Llorà et al., 2008 and Bacardit et al., 2009).

It is important to note that the CCEA and DNFEA algorithms do not necessarily need to be run in tandem, and can each be used independently. For example, Hanley (2017) uses the CCEA alone to mine the Chagas dataset to find a variety of very fit CCs that could be more closely examined by domain experts to assess (a) new insights regarding combinations of risk factors associated with T. dimidiata infestation, and (b) whether these CCs might inform the design of new ecohealth interventions to slow the spread of Chagas disease in a feasible, effective, and cost-effective manner. Similarly, the DNFEA can be used independently of the CCEA (e.g., to identify heterogeneous rule sets comprised of CCs using methods other than the CCEA, such as through LCS, Genetic Programming, Random Chemistry (Eppstein et al., 2007), or even exhaustive search (if the size of the CC search space is small enough). Conversely, if the CCEA archives only a few CCs (as in the synthetic genome problem), one could bypass the DNFEA and simply use exhaustive search on the CCEA archive to identify the causal DNF.

\subsection{Majority-On Problem: LCS versus CCEA-DNFEA}

The presence of overlapping CCs is the primary reason the majority-on problem has been used as a benchmark in the LCS community (Iqbal et al., 2013b,c, 2014). One of the most reliable Michigan-style LCSs, referred to as XCS, is known to struggle with overlap (Kovacs, 2002; Ioannides et al., 2011). Kovacs (2002) noted that XCS penalizes against overlapping CCs; and Ioannides et al. (2011) showed that even when XCS is initialized with a population containing the overlapping true signals, they are selected out of the CC population.

When Iqbal et al. (2013c) used XCS to tackle the 7-bit majority-on problem, the evolved CCs were an order or two below that of the true causal CCs. On the other hand, when an XCS variant was used, one that evolves a logical representation of the action set, the selected CCs were often (23 out of 30 times) at least one order greater than the true causal CCs (Iqbal et al., 2013c). Thus, even when 100\% PPV was reported for small majority-on problems (3-, 5-, and 7-bit) (Iqbal et al., 2013b,c, 2014), the true causal CCs were not identified. This is not surprising, given that our analysis (see Fig. B1) shows many suboptimal CCs with $100 \%$ PPV. Significant overfitting is likely in these 
large populations of overly specific classifiers. Although total evaluations are not explicitly reported in the LCS community, we observe from published plots that reported evaluations for low-order majority-on problems (Iqbal et al., 2013c, 2014) are orders of magnitude larger than the number of possible CCs in the search space.

In contrast, the proposed CCEA consistently and efficiently archived all of the true causal CCs in all majority-on problems tested (up to 11-bit). The DNFEA archived the single true causal DNF in up to 5-bit majority-on problems, and this causal DNF was easily identifiable as the archived clause with the best Fitness. Because the feature sensitivity test cannot be employed for the majority-on problem (see Subsection 3.1), ad hoc postprocessing was required to reduce the size of the CC archive before the DNFEA could be effectively used for 7-bit to 11-bit majority-on problems.

\subsection{Multiplexer Problem: LCS versus CCEA-DNFEA}

The presence of tunable degrees of heterogeneity and epistasis is the primary reason the multiplexer problem continues as a standard benchmark problem in the LCS community. As the size of the multiplexer problem increases, the number of true CCs in the true causal DNF increases (albeit not as rapidly as in the majority-on problem) and the individual coverage rapidly decreases (Table 3). As in previous studies (Kovacs, 1998; Butz et al., 2003), we also found many noncausal CCs with the same PPV and expected coverage as the true causal CCs.

Both Michigan-style and Pittsburgh-style LCSs have been used to tackle the multiplexer problem. Although the lowest number of training instances used by Michiganstyle LCS on the multiplexer problem shown by Iqbal et al. (2013c, 2014) were the same order of magnitude as the number of \#Total Evals reported here, this LCS was not able to directly evolve the true causal CCs even when achieving $100 \%$ prediction accuracy. However, with additional ad hoc post-processing, the true causal DNFs were recovered on balanced multiplexer problems up to 37-bits (Iqbal et al., 2013a). Bacardit and Krasnogor (2009) evolved a nearly-causal rule set for the 37-bit multiplexer problems using a modified Pittsburgh-style LCS with smart crossover (first introduced in Bacardit and Krasnogor, 2006), again using the same order of magnitude number of training instances as \#Total Evals reported here; they report that the causal DNF was identified when the algorithm was allowed to run longer, but did not specify how much longer.

Our proposed approach consistently evolved the single true causal DNF in all multiplexer problems tested (up to 37-bit). Even when we introduced extraneous features, imbalanced classes, noise in the class associations, and missing data into a 6-bit multiplexer problem, our methodology reliably evolved and identified the single true causal 4th-order DNF of 3rd-order CCs (Fig. 2). It is encouraging that the CCEA and DNFEA performed so strongly in the face of significant class imbalance, noise in class associations, and missing data, because these are often characteristics of real-world datasets. Of particular importance is the ability to handle missing data, without the need impute with potentially misleading synthetic data.

\subsection{Synthetic Genome Problem: LCS versus CCEA-DNFEA}

The synthetic genome problem introduced in Urbanowicz and Moore (2010a) was designed as a more realistic dataset representing a heterogeneous, purely epistatic problem, in which the true causal DNF is a 4 th-order disjunction of four 2 nd-order CCs. This dataset includes 16 extraneous features and an imperfect association between the true features and balanced binary outcome classes. 
Urbanowicz and Moore (2010b) report an inability to evolve correct rule sets for this problem when using a Pittsburgh-style LCS. They used the Michigan-style XCS to evolve rules for predicting both class 0 and class 1 (Urbanowicz and Moore, 2010a) and reported an average classification accuracy of over $88 \%$ using 10 -fold cross validation with 1,600 classifiers trained on 1,440 unique training instances (repeatedly sampled for a total of $1,000,000$ instances shown to XCS), and up to $72 \%$ on the testing data. Thus, despite their use of cross-validation there was still evidence of overfitting, since the average training accuracy was over $20 \%$ higher, and the average testing accuracy over $5 \%$ higher, than the accuracy of the true causal rule set.

Our approach consistently archived all 4 true causal CCs and the single true causal DNF, which was readily identifiable as (a) the most parsimonious of the highly fit clauses and (b) had the highest coverage in the resulting DNF archive. This more realistic problem, relative to the majority-on or multiplexer problems, also highlights the importance of using the feature sensitivity test, which reduced the number of CCs passed to the archive by two orders of magnitude.

\subsection{Chagas Vector Infestation Dataset}

Some of the feature interactions evolved by the CCEA on the Chagas vector infestation data had been previously identified as potential drivers of infestation, which increases our confidence in the CCEA results. However, the CCEA analysis also provided ranges of co-evolved values of interacting features that were most strongly associated with infestation as well as new feature interactions not recognized previously. Ongoing analysis of the CCEA and DNFEA Chagas survey results is being used to inform the design of eco-interventions aimed at slowing the spread of Chagas disease. While a full discussion of this application is beyond the scope of this article, we refer the interested reader to Hanley (2017) for more details.

\section{Summary}

We developed a new method, and provide open source code (Hanley, 2019), for identifying parsimonious complex rule sets from batch data that may include features of arbitrary arity and multiple class outcomes.

We use a hypergeometric probability mass function as a principled statistic for assessing fitness of potential causal rules in complex classification problems. This fitness function formally quantifies the probability that an observed association between a rule and a class outcome is due to chance, taking into account the size of the dataset, the amount of missing data, and the distribution of class outcomes. We employ this fitness metric in two back-to-back age-layered evolutionary algorithms. The first evolves an archive of probabilistically significant conjunctive clauses, incorporating co-evolved sets of feature values in these clauses. The second evolves an archive of probabilistically significant disjunctions of the archived conjunctions. Each archive uses separate bins for clauses of different orders, with dynamically adjusted, order-specific fitness and optional feature sensitivity thresholds. The combination of these algorithmic attributes enables our method to discover parsimonious, epistatic, heterogeneous, and overlapping rule sets, even in the presence of imbalanced classes, missing data, and noisy class associations.

By decomposing the problem into first finding good conjunctive clauses and then finding good disjunctions of them, our approach is shown to dramatically reduce the size of the search space (relative to alternative methods that seek to evolve compact rule sets, such as Pittsburgh-style LCSs) and yet returns "white box," potentially causal, 


\section{J. P. Hanley et al.}

parsimonious rule sets (in contrast to Michigan Style LCSs, which return large populations of rules, are prone to overfitting, and may not even contain the true causal rules).

We validated the method on two types of scalable benchmark problems with known causal rule sets (majority-on and multiplexer problems, which include various degrees of heterogeneity, epistasis, and overlap in the rule sets) from datasets where we had introduced extraneous features, class imbalance, noise in class outcomes, and missing data. We also validated on a more realistic noisy synthetic genome problem with purely epistatic and heterogeneous rules. Analyses of the fitness landscapes of these problems illustrate why more ad hoc combinations of PPV and coverage may be insufficient for identifying the true causal rule sets.

In all the epistatic and heterogeneous benchmark problems tested, we consistently evolved the true causal rule sets in the form of a single clause in disjunctive normal form, thus truly solving these problems in the strongest sense of the word. This distinguishes our method from machine learning classifiers whose aim is only to maximize prediction accuracy and coverage on sampled data. To our knowledge, no other method has been shown to consistently identify such complex causal rule sets from datasets with this level of complexity.

Finally, we applied the method to the real-world survey dataset that initially motivated us to develop the method. Our ongoing analysis of the results is providing important practical insights to inform eco-intervention strategies aimed at slowing the spread of the deadly Chagas disease.

\section{Acknowledgments}

This work was supported, in part, by Vermont EPSCoR with funds from the National Science Foundation Grant EEID BCS-1216193 (JPH) and EPS-1101317 (DMR).

\section{References}

Aguilar-Ruiz, J., Riquelme, J., and Toro, M. (2003). Evolutionary learning of hierarchical decision rules. IEEE Transactions on Systems, Man and Cybernetics, Part B (Cybernetics), 33(2):324331.

Bacardit, J., Burke, E. K., and Krasnogor, N. (2009). Improving the scalability of rule-based evolutionary learning. Memetic Computing, 1(1):55-67.

Bacardit, J., and Krasnogor, N. (2006). Smart crossover operator with multiple parents for a Pittsburgh learning classifier system. In Proceedings of the 8th Annual Conference on Genetic and Evolutionary Computation (GECCO), p. 1441.

Bacardit, J., and Krasnogor, N. (2009). Performance and efficiency of memetic Pittsburgh learning classifier systems. Evolutionary Computation, 17(3):307-342.

Barto, A. G. (1985). Learning by statistical cooperation of self-interested neuron-like computing elements. Human Neurobiology, 4(4):229-256.

Booker, L. B. (1989). Triggered rule discovery in classifier systems. In Proceedings of the Third International Conference on Genetic Algorithms, Vol. 3, pp. 265-274.

Breiman, L. (2001). Random forests. Machine Learning, 45(1):5-32.

Bustamante Zamora, D. M., Hernández, M. M., Torres, N., Zúniga, C., Sosa, W., de Abrego, V., and Monroy Escobar, M. C. (2015). Information to act: Household characteristics are predictors of domestic infestation with the Chagas vector Triatoma dimidiata in Central America. American Journal of Tropical Medicine and Hygiene, 93(1):97-107. 
Butz, M., Kovacs, T., Lanzi, P., and Wilson, S. (2004). Toward a theory of generalization and learning in XCS. IEEE Transactions on Evolutionary Computation, 8(1):28-46.

Butz, M. V., Goldberg, D. E., and Tharakunnel, K. (2003). Analysis and improvement of fitness exploitation in XCS: Bounding models, tournament selection, and bilateral accuracy. Evolutionary Computation, 11(3):239-277.

Butz, M. V., Pelikan, M., Llorà, X., and Goldberg, D. E. (2006). Automated global structure extraction for effective local building block processing in XCS. Evolutionary Computation, 14(3):345380.

Butz, M. V., Sastry, K., and Goldberg, D. E. (2005). Strong, stable, and reliable fitness pressure in XCS due to tournament selection. Genetic Programming and Evolvable Machines, 6(1):53-77.

Buxbaum, J. D., Silverman, J. M., Smith, C. J., Kilifarski, M., Reichert, J., Hollander, E., Lawlor, B. A., Fitzgerald, M., Greenberg, D. A., and Davis, K. L. (2001). Evidence for a susceptibility gene for autism on chromosome 2 and for genetic heterogeneity. The American Journal of Human Genetics, 68(6):1514-1520.

Calian, D. A., and Bacardit, J. (2013). Integrating memetic search into the BioHEL evolutionary learning system for large-scale datasets. Memetic Computing, 5(2):95-130.

De Jong, K. A., and Spears, W. M. (1991). Learning concept classification rules using genetic algorithms. In Proceedings of the Twelfth International Joint Conference on Artificial Intelligence, Vol. 12, pp. 651-656.

Eppstein, M. J., and Hines, P. D. H. (2012). A "random chemistry" algorithm for identifying collections of multiple contingencies that initiate cascading failure. IEEE Transactions on Power Systems, 27(3):1698-1705.

Eppstein, M. J., Payne, J. L., White, B. C., and Moore, J. H. (2007). Genomic mining for complex disease traits with random chemistry. Genetic Programming and Evolvable Machines, 8(4):395411.

Franco, M. A., Krasnogor, N., and Bacardit, J. (2011). Modelling the initialisation stage of the ALKR representation for discrete domains and GABIL encoding. In Proceedings of the 13th Annual Conference on Genetic and Evolutionary Computation (GECCO), pp. 1291-1298.

Franco, M. A., Krasnogor, N., and Bacardit, J. (2012). Analysing BioHEL using challenging Boolean functions. Evolutionary Intelligence, 5(2):87-102.

Goldberg, D. E. (1989). Genetic algorithms in search, optimization, and machine learning. Reading, MA: Addison-Wesley.

Hanley, J. P. (2017). A new evolutionary algorithm for mining noisy, epistatic, geospatial survey data associated with Chagas disease. PhD thesis, Faculty of the Graduate College, The University of Vermont.

Hanley, J. P. (2019). MATLAB code available at https://www.mathworks.com/matlabcentral /fileexchange/69950-ccea-and-dnfea.

Hanley, J. P., Eppstein, M. J., Buzas, J. S., and Rizzo, D. M. (2016). Evolving probabilistically significant epistatic classification rules for heterogeneous big datasets. In Proceedings of the 18th Annual Conference on Genetic and Evolutionary Computation, pp. 445-452.

Holland, J. H., and Reitman, J. S. (1978). Cognitive systems based on adaptive algorithms. In An overview of pattern-directed inference systems, pp. 313-329. Rand Corporation, Santa Monica, CA.

Hornby, G. S. (2006). ALPS: The age-layered population structure for reducing the problem of premature convergence. In Proceedings of the 8th Annual Conference on Genetic and Evolutionary Computation, p. 815. 
Huang, G. Q., Li, L., and Chen, X. (2007). A tandem evolutionary algorithm for platform product customization. Journal of Computing and Information Science in Engineering, 7(2):151-159.

Ioannides, C., Barrett, G., and Eder, K. (2011). XCS cannot learn all Boolean functions. In Proceedings of the 13th Annual Conference on Genetic and Evolutionary Computation, p. 1283.

Iqbal, M., Browne, W. N., and Mengjie Zhang (2014). Reusing building blocks of extracted knowledge to solve complex, large-scale Boolean problems. IEEE Transactions on Evolutionary Computation, 18(4):465-480.

Iqbal, M., Browne, W. N., and Zhang, M. (2012). Extracting and using building blocks of knowledge in learning classifier systems. In Proceedings of the 14th Annual Conference on Genetic and Evolutionary Computation, p. 863.

Iqbal, M., Browne, W. N., and Zhang, M. (2013a). Evolving optimum populations with XCS classifier systems: XCS with code fragmented action. Soft Computing, 17(3):503-518.

Iqbal, M., Browne, W. N., and Zhang, M. (2013b). Extending learning classifier system with cyclic graphs for scalability on complex, large-scale Boolean problems. In Proceedings of the 15th Annual Conference on Genetic and Evolutionary Computation, p. 1045.

Iqbal, M., Browne, W. N., and Zhang, M. (2013c). Learning complex, overlapping and niche imbalance Boolean problems using XCS-based classifier systems. Evolutionary Intelligence, 6(2):7391.

Iqbal, M., Browne, W. N., and Zhang, M. (2015). Improving genetic search in XCS-based classifier systems through understanding the evolvability of classifier rules. Soft Computing, 19(7):1863-1880.

Jarlenski, M., Hyon Baik, S., and Zhang, Y. (2016). Trends in use of medications for smoking cessation in Medicare, 2007-2012. American Journal of Preventive Medicine, 51(3):301-308.

Kaplinski, M., Jois, M., Galdos-Cardenas, G., Rendell, V. R., Shah, V., Do, R. Q., Marcus, R., Burroughs Pena, M. S., del Carmen Abastoflor, M., LaFuente, C., Bozo, R., Valencia, E., Verastegui, M., Colanzi, R., Gilman, R. H., and Bern, C. (2015). Sustained domestic vector exposure is associated with increased Chagas cardiomyopathy risk but decreased parasitemia and congenital transmission risk among young women in Bolivia. Clinical Infectious Diseases, 61(6):918-926.

Kendall, M. G. (1952). The advanced theory of statistics, volume 1. 3rd ed. New York: Hafner Publishing Company.

Kirino, Y., Bertsias, G., Ishigatsubo, Y., Mizuki, N., Tugal-Tutkun, I., Seyahi, E., Ozyazgan, Y., Sacli, F. S., Erer, B., Inoko, H., et al. (2013). Genome-wide association analysis identifies new susceptibility loci for Behcet's disease and epistasis between hla-b [ast] 51 and erap1. Nature Genetics, 45(2):202-207.

Kovacs, T. (1998). XCS classifier system reliably evolves accurate, complete, and minimal representations for Boolean functions. In P. K. Chawdhry, R. Roy, and R. K. Pant (Eds.), Soft computing in engineering design and manufacturing, pp. 59-68. London: Springer London.

Kovacs, T. (2002). What should a classifier system learn and how should we measure it? Soft Computing-A Fusion of Foundations, Methodologies and Applications, 6(3-4):171-182.

Larivière, S. (2001). Ursus americanus. Mammalian Species, 647:1-11.

LeCun, Y., Bengio, Y., and Hinton, G. (2015). Deep learning. Nature, 521(7553):436.

Li, K., Simons-Morton, B., Gee, B., and Hingson, R. (2016). Marijuana-, alcohol-, and drugimpaired driving among emerging adults: Changes from high school to one-year post-high school. Journal of Safety Research, 58:15-20. 
Llorà, X., Sastry, K., and Goldberg, D. (2005). The compact classifier system: Scalability analysis and first results. In The 2005 IEEE Congress on Evolutionary Computation, Vol. 1, pp. 596-603.

Llorà, X., Sastry, K., Lima, C. F., Lobo, F. G., and Goldberg, D. E. (2008). Linkage learning, rule representation, and the X-ray extended compact classifier system. In 10th International Workshop, pp. 189-205.

Markellos, R. N., Psychoyios, D., and Schneider, F. (2016). Sovereign debt markets in light of the shadow economy. European Journal of Operational Research, 252(1):220-231.

McDermott, J., De Jong, K., O’Reilly, U.-M., White, D. R., Luke, S., Manzoni, L., Castelli, M., Vanneschi, L., Jaskowski, W., Krawiec, K., and Harper, R. (2012). Genetic programming needs better benchmarks. In Proceedings of the 14th Annual Conference on Genetic and Evolutionary Computation, p. 791.

Molina, I., Salvador, F., Sánchez-Montalvá, A., Trevi no, B., Serre, N., Sao Avilés, A., and Almirante, B. (2015). Toxic profile of Benznidazole in patients with chronic Chagas disease: Risk factors and comparison of the product from two different manufacturers. Antimicrobial Agents and Chemotherapy, 59(10):6125-6131.

Moore, J. H. (2003). The ubiquitous nature of epistasis in determining susceptibility to common human diseases. Human Heredity, 56(1-3):73-82.

Nesheli, M. M., Ceder, A. A., and Estines, S. (2016). Public transport user's perception and decision assessment using tactic-based guidelines. Transport Policy, 49:125-136.

Nuzzo, R. (2014). Scientific method: Statistical errors. Nature, 506(7487):150-152.

Olivera, M. J., Cucunuba, Z. M., Alvarez, C. A., and Nicholls, R. S. (2015). Safety profile of Nifurtimox and treatment interruption for chronic Chagas disease in Colombian adults. American Journal of Tropical Medicine and Hygiene, 93(6):1224-1230.

Poole, K. T., and Rosenthal, H. (1984). The polarization of American politics. The Journal of Politics, 46(4):1061-1079.

Rapp, J. P., Garrett, M. R., and Deng, A. Y. (1998). Construction of a double congenic strain to prove an epistatic interaction on blood pressure between rat chromosomes 2 and 10. Journal of Clinical Investigation, 101(8):1591-1595.

Ritchie, M. D., Hahn, L. W., Roodi, N., Bailey, L. R., Dupont, W. D., Parl, F. F., and Moore, J. H. (2001). Multifactor-dimensionality reduction reveals high-order interactions among estrogen-metabolism genes in sporadic breast cancer. The American Journal of Human Genetics, 69(1):138-147.

Smith, S. F. (1980). A learning system based on genetic adaptive algorithms. PhD thesis, University of Pittsburgh.

Urbanowicz, R. J., Andrew, A. S., Karagas, M. R., and Moore, J. H. (2013). Role of genetic heterogeneity and epistasis in bladder cancer susceptibility and outcome: A learning classifier system approach. Journal of the American Medical Informatics Association, 20(4):603-612.

Urbanowicz, R. J., Bertasius, G., and Moore, J. (2014). ExSTraCS 1.0: An extended Michigan-style learning classifier system for flexible supervised learning. In Parallel Problem Solving from Nature, pp. 211-221.

Urbanowicz, R. J., and Moore, J. H. (2009). Learning classifier systems: A complete introduction, review, and roadmap. Journal of Artificial Evolution and Applications, 2009:1-25.

Urbanowicz, R. J., and Moore, J. H. (2010a). The application of Michigan-style learning classifier systems to address genetic heterogeneity and epistasis in association studies. In Proceedings of the 12th Annual Conference on Genetic and Evolutionary Computation, p. 195. 
J. P. Hanley et al.

Urbanowicz, R. J., and Moore, J. H. (2010b). The application of Pittsburgh-style learning classifier systems to address genetic heterogeneity and epistasis in association studies. In International Conference on Parallel Problem Solving from Nature, pp. 404-413.

Urbanowicz, R. J., and Moore, J. H. (2015). ExSTraCS 2.0: Description and evaluation of a scalable learning classifier system. Evolutionary Intelligence, 8(2-3):89-116.

Wilson, I. D. (2009). Drugs, bugs, and personalized medicine: Pharmacometabonomics enters the ring. Proceedings of the National Academy of Sciences, 106(34):14187-14188.

Wilson, N. L., Rickard, B. J., Saputo, R., and Ho, S.-T. (2017). Food waste: The role of date labels, package size, and product category. Food Quality and Preference, 55:35-44.

Wilson, S. W. (1987a). Classifier systems and the animat problem. Machine Learning, 2(3):199-228.

Wilson, S. W. (1987b). Quasi-Darwinian learning in a classifier system. In Proceedings of the Fourth International Machine Learning Workshop, Vol. 4, pp. 59-65.

Wilson, S. W. (1995). Classifier fitness based on accuracy. Evolutionary Computation, 3(2):149-175.

Wu, X., Zhu, X., Wu, G.-Q., and Ding, W. (2014). Data mining with big data. IEEE Transactions on Knowledge and Data Engineering, 26(1):97-107.

Young Kim, E., and Kim, Y. (2004). Predicting online purchase intentions for clothing products. European Journal of Marketing, 38(7):883-897.

Yousefi, S., Moradi, H., Boll, J., and Schönbrodt-Stitt, S. (2016). Effects of road construction on soil degradation and nutrient transport in Caspian Hyrcanian mixed forests. Geoderma, 284:103112. 